IAC-04-S.P.11

\title{
SOLAR SAIL PERFORMANCE REQUIREMENTS FOR MISSIONS TO THE OUTER SOLAR SYSTEM AND BEYOND
}

\author{
Bernd Dachwald \\ German Aerospace Center (DLR), Institute of Space Simulation, Linder Hoehe, 51147 Cologne \\ bernd.dachwald@dlr.de
}

\begin{abstract}
Solar sails enable missions to the outer solar system and beyond, although the solar radiation pressure decreases with the square of solar distance. For such missions, the solar sail may gain a large amount of energy by first making one or more close approaches to the sun. Within this paper, optimal trajectories for solar sail missions to the outer planets and into near interstellar space (200 AU) are presented. Thereby, it is shown that even near/medium-term solar sails with relatively moderate performance allow reasonable transfer times to the boundaries of the solar system.
\end{abstract}

\section{INTRODUCTION}

Utilizing solely the freely available solar radiation pressure for propulsion, solar sails make a wide range of high- $\Delta V$ missions feasible. They enable even missions to the outer solar system and beyond, despite the fact that the solar radiation pressure decreases with the square of the sun-sail distance. SAUER observed that the solar sail may gain a large amount of energy by making a close approach to the sun, turning the trajectory into a hyperbolic one [1], a maneuver for which LEIPOLD coined the term 'solar photonic assist' (SPA) [2, 3]. SAUER made parametric studies for near-interstellar missions to $100 \mathrm{AU}, 250 \mathrm{AU}$ and $1000 \mathrm{AU}$ using ideal ${ }^{1}$ very-high-performance solar sails that perform only a single SPA [4]. LEIPOLD observed that solar sails with a more conservative performance require $\mathrm{mul}$ tiple SPAs to achieve solar system escape. He gave some (non-optimized) example trajectories for ideal solar sails but made no parametric studies.

Within this paper, optimal trajectories for solar sail missions to the outer planets and into near interstellar space (200 AU) are presented, both for ideal and for non-ideal ${ }^{2}$ sails. It will be shown that even for near/medium-term solar sails with relatively moderate performance - multiple SPA trajectories allow reasonable transfer times (without the need to perform any gravity assist maneuver).

\footnotetext{
${ }^{1}$ where the incident radiation is reflected specularly

2 where a fraction of the incoming radiation is absorbed and a fraction of the reflected radiation is reflected nonspecularly
}

Nevertheless, without the use of additional propulsive devices and/or an aerocapture maneuver at the target, only fast flybys can be accomplished due to the associated large hyperbolic excess velocities.

For the calculation of near-globally optimal trajectories, evolutionary neurocontrol is used, a novel method that is based on artificial neural networks and evolutionary algorithms $[5,6,7]$.

\section{SOLAR SAIL FORCE MODELS}

For describing the solar radiation pressure (SRP) force exerted on a solar sail, it is convenient to introduce two unit vectors. The first one is the sail normal vector $\mathbf{n}$, which is perpendicular to the sail surface and always directed away from the sun. Its direction, which describes the sail attitude, is expressed by the sail clock angle $\alpha$ and the sail cone angle $\beta$ (Fig. 1).

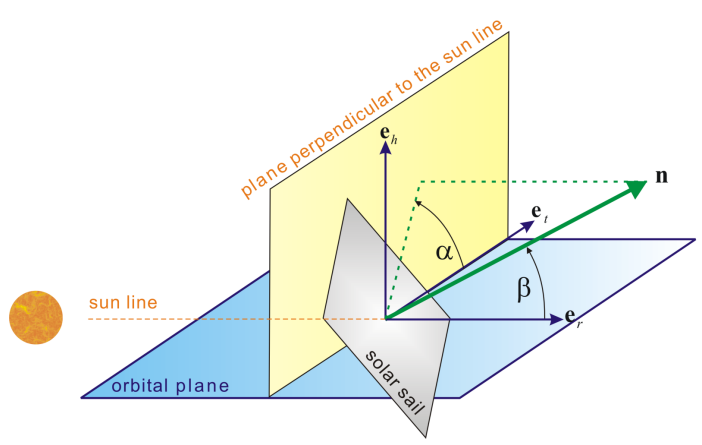

Fig. 1: Definition of the sail normal vector 
The second unit vector is the thrust unit vector f, which points always along the direction of the SRP force. Its direction is described likewise by the thrust clock angle $\gamma$ and the thrust cone angle $\delta$ (Fig. 2).

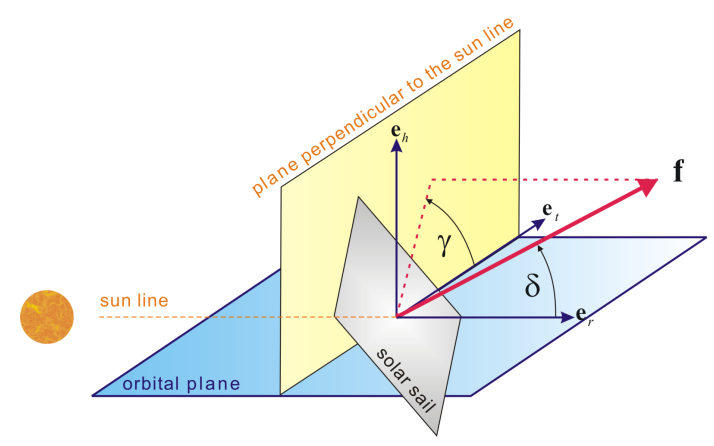

Fig. 2: Definition of the thrust vector

At a distance $r$ from the sun, the SRP is

$$
P=\frac{S_{0}}{c}\left(\frac{r_{0}}{r}\right)^{2}=4.563 \frac{\mu \mathrm{N}}{\mathrm{m}^{2}} \cdot\left(\frac{r_{0}}{r}\right)^{2}
$$

where $S_{0}=1368 \mathrm{~W} / \mathrm{m}^{2}$ is the solar constant, $c$ is the speed of light in vacuum, and $r_{0}=1 \mathrm{AU}$.

For the optical characteristics of a solar sail, different assumptions can be made, which result in different models for the magnitude and direction of the SRP force acting on the sail. The most simple model assumes a specularly reflecting sail surface. The SRP force on such an ideal sail of area $A$ is

$$
\mathbf{F}_{\mathrm{SRP}}=2 P A \cos ^{2} \beta \mathbf{n}
$$

Thus, the SRP force it is always along the direction of the sail normal vector, $\mathbf{f}=\mathbf{n}$.

Another SRP force model that is widely encountered uses an overall sail efficiency factor $\eta \leq 1$ with the intention of describing the non-ideal reflectivity of the sail. Using this factor, the SRP force acting on the sail is

$$
\mathbf{F}_{\mathrm{SRP}}=2 \eta P A \cos ^{2} \beta \mathbf{n}
$$

Also for this model, the SRP force is always along the direction of the sail normal vector, $\mathbf{f}=\mathbf{n}$. Therefore, this model also describes a specularly reflecting sail surface, where the angle of incidence is equal to the angle of reflection. From the perspective of trajectory analysis, this models is equivalent to the ideal sail model because a decrease of $\eta$ can always be offset with an inversely proportional increase of $A$. Therefore, this model is not further considered here.
Because the surface of a real solar sail is not a specular reflector, a thorough trajectory simulation must consider the optical characteristics of the real sail film, as they can be parameterized by the absorption coefficient $\alpha$, the reflection coefficient $\rho$, the transmission coefficient $\tau$, and the emission coefficient $\varepsilon$, with the constraint $\alpha+\rho+\tau=$ 1. The reflection coefficient can be further divided into a coefficient for specular reflection $\rho_{s}$, a coefficient for diffuse reflection $\rho_{d}$, and a coefficient for back reflection $\rho_{b}$, with the constraint $\rho_{s}+\rho_{d}+\rho_{b}=\rho$. The non-ideal solar sail force model used within this paper considers the optical parameters $\mathcal{P}=\left\{\alpha, \rho_{s}, \rho_{d}, \rho_{b}, \tau, \varepsilon_{\mathrm{f}}, \varepsilon_{\mathrm{b}}\right\}$ of a sail film that is aluminum-coated on the front side (emissivity $\varepsilon_{\mathrm{f}}=0.05$ ) and chromium-coated on the back side (emissivity $\varepsilon_{\mathrm{b}}=0.55$ ) to keep the sail temperature within moderate limits $^{3}$. Using the non-ideal solar sail force model, the SRP force has a component

$$
F_{\perp}=2 P A q_{\perp}(\beta, \mathcal{P})
$$

perpendicular to the sail surface and a component

$$
F_{\|}=2 P A q_{\|}(\beta, \mathcal{P})
$$

parallel to the sail surface.

Using the optical parameters for an $\mathrm{Al} \mid \mathrm{Cr}$-coated sail [8], one gets

$$
\begin{aligned}
q_{\perp}\left(\beta, \mathcal{P}_{\mathrm{Al} \mid \mathrm{Cr}}\right) & =0.9136 \cos ^{2} \beta-0.005444 \cos \beta \\
q_{\|}\left(\beta, \mathcal{P}_{\mathrm{Al} \mid \mathrm{Cr}}\right) & =0.0864 \sin \beta \cos \beta
\end{aligned}
$$

The SRP force may be written as

$$
\mathbf{F}_{\mathrm{SRP}}=\sqrt{F_{\perp}^{2}+F_{\|}^{2}} \mathbf{f}
$$

and by defining

$$
Q^{2}(\beta, \mathcal{P})=\sqrt{q_{\perp}^{2}(\beta, \mathcal{P})+q_{\|}^{2}(\beta, \mathcal{P})}
$$

as

$$
\mathbf{F}_{\mathrm{SRP}}=2 P A Q^{2}(\beta, \mathcal{P}) \mathbf{f}
$$

Thus, the SRP force is not along the direction of the sail normal vector (except for $\beta=0$ ). The angle between $\mathbf{n}$ and $\mathbf{f}$ is

$$
\varepsilon=\arctan \left(q_{\|} / q_{\perp}\right)
$$

\section{SOLAR SAIL ORBITAL DYNAMICS}

The orbital dynamics of solar sails is in many respects similar to the orbital dynamics of other

\footnotetext{
${ }^{3}$ as it will become clear later from equation (4)
} 
low-thrust spacecraft. Other low-thrust spacecraft, however, may orient its thrust vector into any desired direction, whereas the thrust vector of a solar sail is constrained to lie on the surface of a bubble that is always directed away from the sun (Fig. 3). Nevertheless, by controlling the sail orientation relative to the sun, a solar sail can gain orbital angular momentum and spiral outwards - away from the sun - or lose orbital angular momentum and spiral inwards - towards the sun.

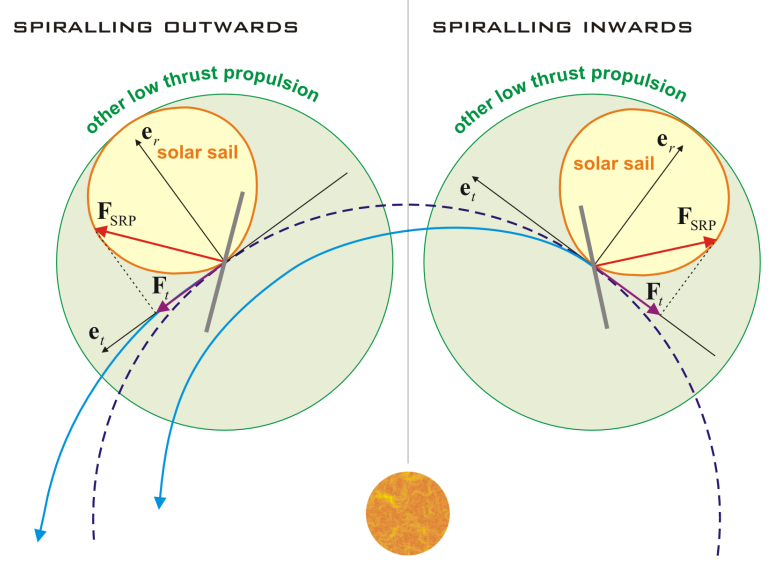

Fig. 3: Spiralling towards and away from the sun

For SPA trajectories, the minimal flight time depends not only on the lightness of the solar sail but also on the minimal solar distance along the trajectory (see Fig. 5). The smaller the minimal solar distance, the larger the amount of energy that can be gained during a SPA. The sail's equilibrium temperature at a distance $r$ from the sun is

$$
T=\left(\frac{1-\rho}{\varepsilon_{\mathrm{f}}+\varepsilon_{\mathrm{b}}} \frac{S_{0}}{\sigma} \frac{r_{0}^{2}}{r^{2}} \cos \beta\right)^{1 / 4} \propto \frac{\cos ^{1 / 4} \beta}{r^{1 / 2}}
$$

where $\sigma$ is the STEFAn-BoltzMAnN constant [9]. Therefore, the minimal distance to the sun is - for a given sail attitude - limited by the temperature limit of the sail film.

Trajectory optimization for SPA trajectories is exceedingly difficult because one must not only beware of flying too close to the sun but one must also take care that the trajectory becomes not hyperbolic too early, so that no additional energy can be gained. At the same time, one must find the optimal trade-off between the time that is spent within the inner solar system - to gain energy - and the time that is required to fly outwards after the trajectory became hyperbolic.

\section{SOLAR SAIL PERFORMANCE PARAMETERS}

The following performance parameters are commonly used to describe the lightness of solar sails:

The sail assembly loading is defined as the mass of the sail assembly (the sail film and the required structure for storing, deploying and tensioning the sail, index 'SA') per unit area:

$$
\sigma_{\mathrm{SA}}=\frac{m_{\mathrm{SA}}}{A}
$$

The sail assembly loading is the key parameter for the efficiency of the solar sail's structural design.

The sailcraft loading, the key parameter for the lightness of the entire solar sailcraft, is defined as the specific mass of the sailcraft including the payload (index 'PL'), where the term payload stands for the total sailcraft except the solar sail assembly (i.e., except the propulsion system):

$$
\sigma=\frac{m}{A}=\frac{m_{\mathrm{SA}}+m_{\mathrm{PL}}}{A}=\sigma_{\mathrm{SA}}+\frac{m_{\mathrm{PL}}}{A}
$$

The characteristic acceleration is an equivalent parameter for expressing the lightness of the entire solar sailcraft. It is defined as the SRP acceleration acting on a solar sail that is oriented perpendicular to the sun-line at $1 \mathrm{AU}$ :

$$
\begin{aligned}
a_{c} & =\frac{2 S_{0} / c \cdot A \cdot q_{\perp}(0, \mathcal{P})}{m}=\frac{P_{\mathrm{eff}, 0}(\mathcal{P}) \cdot A}{m} \\
& =\frac{P_{\mathrm{eff}, 0}(\mathcal{P})}{\sigma_{\mathrm{SA}}+m_{\mathrm{PL}} / A}
\end{aligned}
$$

For an Al|Cr-coated sail, $P_{\text {eff }, 0}\left(\mathcal{P}_{\mathrm{Al} \mid \mathrm{Cr}}\right)=2 S_{0} / c$. $q_{\perp}\left(0, \mathcal{P}_{\mathrm{Al} \mid \mathrm{Cr}}\right)=8.288 \mu \mathrm{N} / \mathrm{m}^{2}$.

\section{SOLAR SAIL TRAJECTORY OPTIMIZATION}

Within this paper, evolutionary neurocontrol (ENC) is used for the calculation of near-globally optimal trajectories. This method is based on artificial neural networks (ANNs) and evolutionary algorithms (EAs). ENC attacks trajectory optimization problems from the perspective of artificial intelligence and machine learning. Here, it can only be sketched how this method is used to search optimal solar sail trajectories. The reader who is interested in the details of the method is referred to Refs. [5, 6, 7].

The problem of searching an optimal solar sail trajectory $\mathbf{x}^{\star}[t]=\left(\mathbf{r}^{\star}[t], \dot{\mathbf{r}}^{\star}[t]\right)$, where ' $\left.[t]\right]^{\prime}$ denotes the time history of the preceding variable, is equivalent to the problem of searching an optimal sail 
normal vector history $\mathbf{n}^{\star}[t]$, as it is defined by the optimal time history of the so-called direction unit vector $\mathbf{d}^{\star}[t]$, a unit vector that points along the optimal thrust direction. Within the context of machine learning, a trajectory is regarded as the result of a sail steering strategy $S$ that maps the problem relevant variables (the solar sail state $\mathbf{x}$ and the target state $\mathbf{x}_{\mathrm{T}}$ ) onto the direction unit vector, $\mathrm{S}:\left\{\mathbf{x}, \mathbf{x}_{\mathrm{T}}\right\} \subset \mathbb{R}^{12} \mapsto\{\mathbf{d}\} \subset \mathbb{R}^{3}$, from which $\mathbf{n}$ is calculated. This way, the problem of searching $\mathbf{x}^{\star}[t]$ is equivalent to the problem of searching (or learning) the optimal sail steering strategy $\mathrm{S}^{\star}$. An ANN may be used as a so-called neurocontroller (NC) to implement solar sail steering strategies. It can be regarded as a parameterized function $N_{\boldsymbol{\pi}}$ (the network function) that is - for a given network topology - completely defined by the internal parameter set $\boldsymbol{\pi}$ of the ANN. Therefore, each $\boldsymbol{\pi}$ defines a sail steering strategy $S_{\boldsymbol{\pi}}$. The problem of searching $\mathbf{x}^{\star}[t]$ is therefore equivalent to the problem of searching the optimal NC parameter set $\pi^{\star}$. EAs that work on a population of strings can be used for finding $\boldsymbol{\pi}^{\star}$ because $\boldsymbol{\pi}$ can be mapped onto a string $\boldsymbol{\xi}$ (also called chromosome or individual). The trajectory optimization problem is solved when the optimal chromosome $\boldsymbol{\xi}^{\star}$ is found. Fig. 4 sketches the subsequent transformation of a chromosome into a solar sail trajectory. An evolutionary neurocontroller (ENC) is a NC that employs an EA for learning (or breeding) the optimal sail steering strategy.

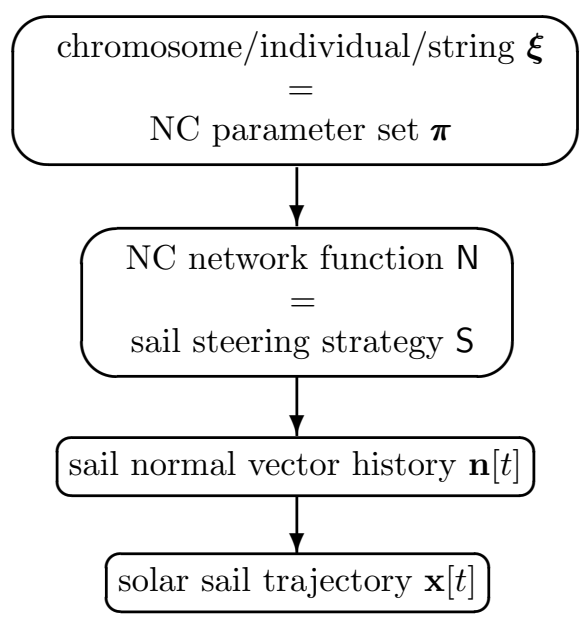

Fig. 4: Transformation of a chromosome into a solar sail trajectory

ENC was implemented by the author within a low-thrust trajectory optimization program called InTrance, which stands for Intelligent Trajectory optimization using neurocontroller evolution. InTrance is a smart global trajectory optimization method that requires only the target body/state and intervals for the initial conditions as input to find a near-globally optimal trajectory for the specified problem. It works without an initial guess and does not require the attendance of a trajectory optimization expert.

\section{RESULTS}

The gravitational forces of all celestial bodies, the SRP force, and disturbing forces are influencing the motion of solar sails. Ideally, all these forces have to be considered for a thorough mission analysis. For mission feasibility analysis, however, as it is done within this paper, the following simplifications are valid: (1) the solar sail is moving under the sole influence of solar gravitation and radiation, (2) the sun is a point mass and a point light source, (3) the solar sail attitude can be changed instantaneously, and (4) the optical characteristics of the sail film do not degrade over time.

All trajectories calculated within this paper assume direct interplanetary insertion of the solar sail with zero hyperbolic excess energy $\left(C_{3}=0 \mathrm{~km}^{2} / \mathrm{s}^{2}\right)$. To find the absolute flight time minima - independent of the actual constellation of Earth and the respective target - no flyby at the target itself but only a crossing of its orbit within a distance of less than $10^{6} \mathrm{~km}$ is required, and InTrance is allowed to vary the launch date within a one year interval. Therefore, the resulting flight times represent lower bounds that are strictly valid only for the optimal constellation of Earth and the respective target. Specific suboptimal launch dates/constellations might yield much longer flight times.

Before calculating minimal flight times for missions to all outer planets and into near interstellar space (200 AU), a Neptune flyby mission will be used to assess the general features of SPA trajectories, to compare different solar sail force models (ideal vs. non-ideal solar sails), and to compare different optimization constraints (limitation of allowed minimal solar distance vs. limitation of allowed maximal sail temperature).

\section{Dependency of Minimal Flight Time on Lightness and Minimal Solar Distance for Ideal Solar Sails}

For a Neptune flyby, InTrance was used to calculate minimal flight times for ideal solar sails with different characteristic accelerations and different solar distance limits. Fig. 5 shows the results. As expected, the trajectories are faster for lighter solar 


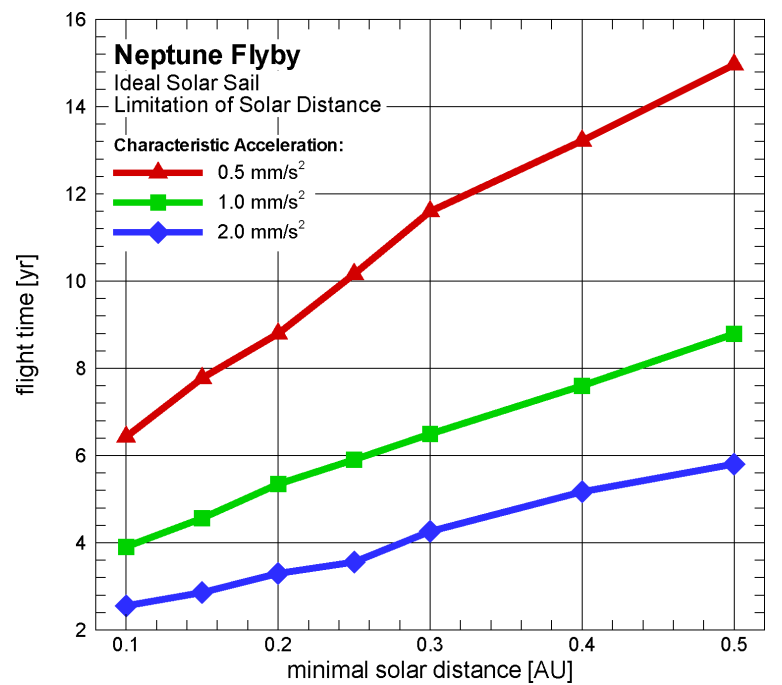

Fig. 5: Flight time over minimal solar distance for different characteristic accelerations

sails and for sails that are allowed to make closer approaches to the sun. Interestingly, the flight time obeys approximately a linear law for all three values of $a_{c}$. Figs. 7 and 8 show optimal Neptune flyby trajectories for four different characteristic accelerations, the allowed minimal solar distance being limited to $0.1 \mathrm{AU}$ in all four cases. One can see that more and more SPAs are required as the characteristic acceleration of the sail decreases.

\section{Ideal vs. Non-Ideal Solar Sails}

In the previous section, minimal flight times have been presented for ideal solar sails because, to the authors knowledge, all previous solar sail trajectory analyses for solar system escape missions assume ideal reflectivity of the sail. A real solar sail, however, is not an ideal reflector and a thorough trajectory analysis must take into account the optical characteristics of the real sail film [10]. In Fig. 6, flight times are compared for an ideal and a nonideal solar sail with $a_{c}=1.0 \mathrm{~mm} / \mathrm{s}^{2}$. Fig. 6 shows that the flight times are about $5 \%$ longer, if the nonideal reflectivity of the sail is taken into account.

\section{Distance-Limited vs. Temperature-Limited Trajectories}

From now on, it will become necessary to distinguish trajectories, for which the allowed minimal solar distance was constrained, from trajectories, for which the allowed maximal sail film temperature was constrained. Therefore, let $r_{\text {lim }}$ denote the allowed minimal solar distance (distance limit) and

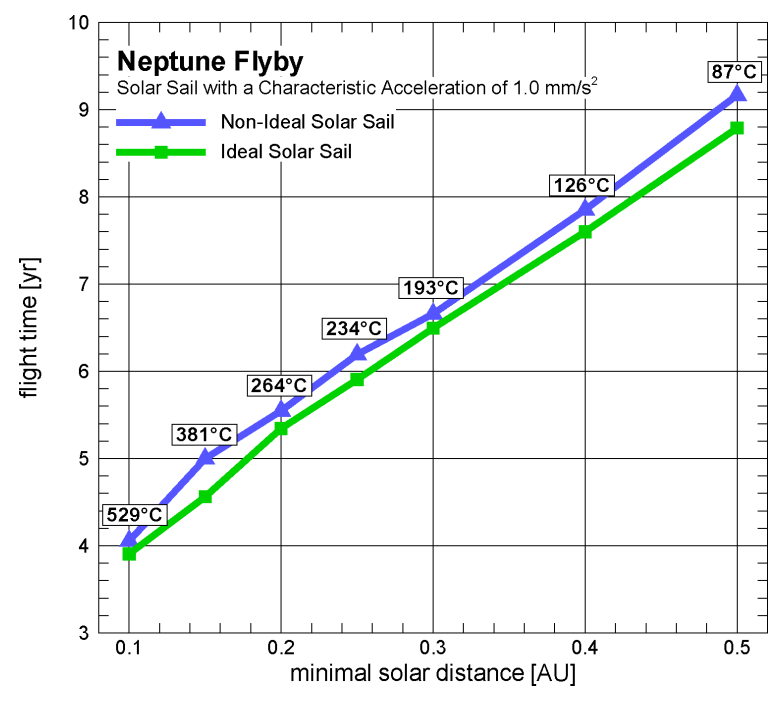

Fig. 6: Comparison of minimal flight times for ideal and non-ideal solar sails

$r_{\text {min }}$ the minimal solar distance along the trajectory, and let $T_{\mathrm{lim}}$ denote the allowed maximal sail film temperature (sail temperature limit) and $T_{\max }$ the maximal sail film temperature along the trajectory. Using this notation, $T_{\max }=T_{\max }\left(r_{\lim }\right)$ (for distance-limited trajectories) and $r_{\min }=r_{\min }\left(T_{\text {lim }}\right)$ (for temperature-limited trajectories).

In previous papers by LeIPOLD [2, 3] and SAUER [4], $r_{\text {lim }}$ was used as a path constraint for the trajectory optimization of ideal solar sails, with the argument that such a constraint enforces that some $T_{\text {lim }}$ will not be exceeded during the closest solar approach. ${ }^{4}$ According to equation (4), however, the temperature at a given solar distance $r$ depends also on the light incidence angle $\beta$. It might therefore yield faster trajectories, if not $r_{\lim }$ but $T_{\lim }$ is directly used as a path constraint. This can be realized by constraining the sail attitude so that the light incidence angle can not become smaller than the critical one, $\beta>\beta_{\lim }\left(r, T_{\lim }\right)$, where $T_{\lim }$ would be exceeded. Fig. 9 shows two exemplary trajectories, one distance-limited (Fig. 9(a)) and one temperature-limited (Fig. 9(b)).

By keeping $\beta$ large enough during the closest approach, the temperature-limited trajectory approaches the sun closer while maintaining the sail temperature below the temperature that is given by the distance-limited trajectory (so that $T_{\max }$ is the same for both trajectories). The resulting trajectory is faster than the distance-limited one. Fig. 10 shows that for a given $T_{\text {lim }}$, optimal temperaturelimited trajectories are on average about $5 \%$ faster

\footnotetext{
${ }^{4}$ although - strictly - the temperature of an ideal sail is always $0 \mathrm{~K}$ according to equation (4)
} 


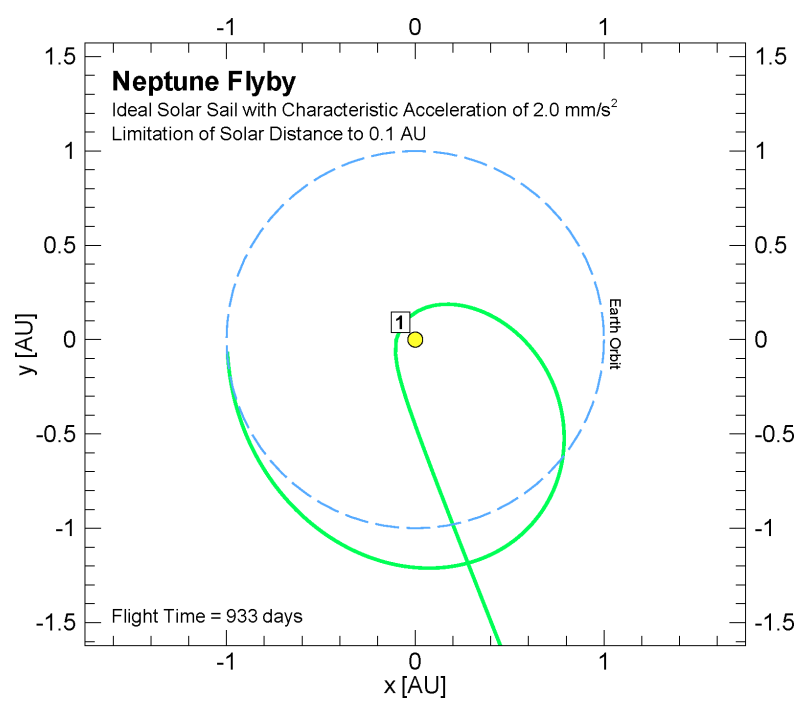

(a) $a_{c}=2.0 \mathrm{~mm} / \mathrm{s}^{2}$, one solar photonic assist

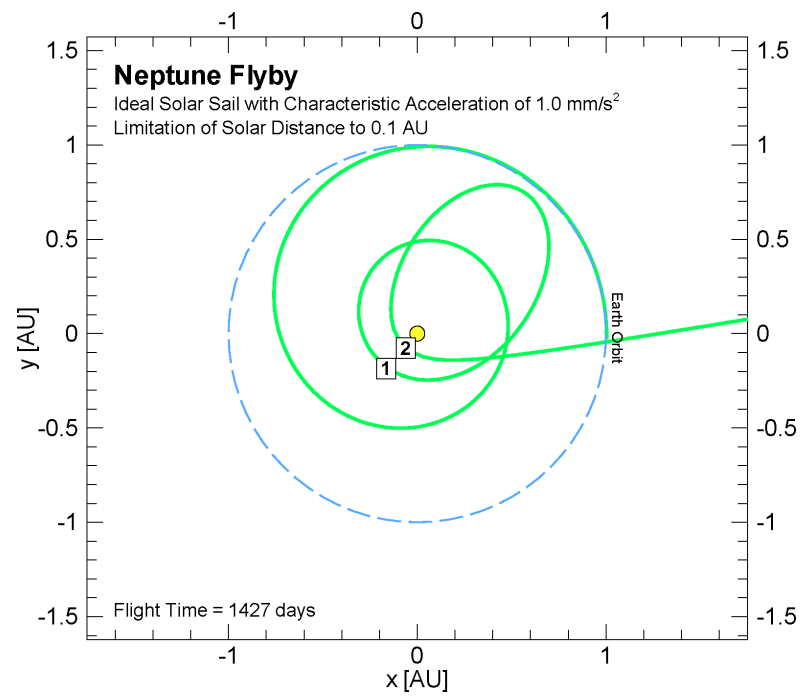

(b) $a_{c}=1.0 \mathrm{~mm} / \mathrm{s}^{2}$, two solar photonic assists

Fig. 7: Topology of optimal Neptune flyby trajectories for different characteristic accelerations

than optimal distance-limited ones. Interestingly, the flight time and and the maximal sail temperature are nearly inversely proportional for both curves.

\section{Minimal Flight Times to the Outer Planets and Near Interstellar Space}

Fig. 11 shows the minimal flight times for optimal temperature-limited flyby trajectories to the outer planets using a non-ideal solar sail. The sail film temperature was limited to $T_{\text {lim }}=240^{\circ} \mathrm{C}$. Interestingly, the flight time obeys nearly a potential law for all targets. Even near-term solar sails

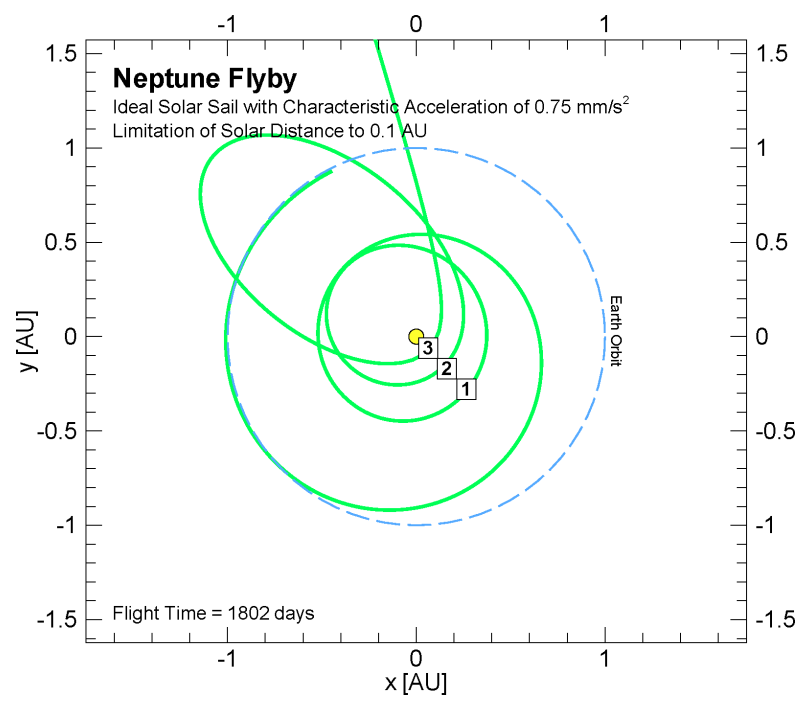

(a) $a_{c}=0.75 \mathrm{~mm} / \mathrm{s}^{2}$, three solar photonic assists

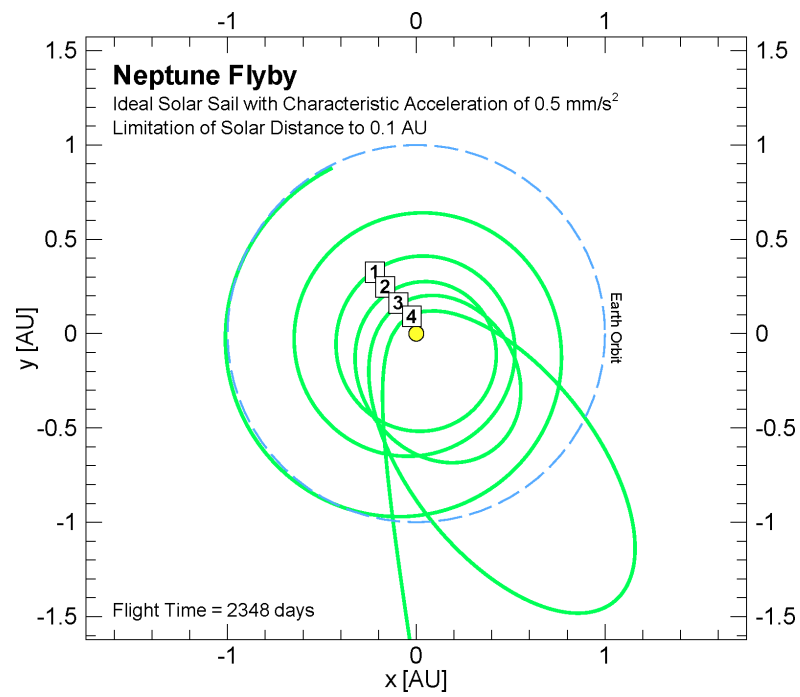

(b) $a_{c}=0.5 \mathrm{~mm} / \mathrm{s}^{2}$, four solar photonic assists

Fig. 8: Topology of optimal Neptune flyby trajectories for different characteristic accelerations

$\left(a_{c} \approx 0.4 \mathrm{~mm} / \mathrm{s}^{2}\right)$ are able to reach Uranus within less than 10 years, and even medium-term solar sails $\left(a_{c} \approx 0.6 \mathrm{~mm} / \mathrm{s}^{2}\right)$ are able to reach Neptune and the inner EDGEWORTH-KUIPER belt within less than 10 years.

\section{Dependency of Minimal Flight Time on Sail Temperature Limit for Non-Ideal Solar Sails}

Fig. 12 shows for three different sail temperature limits $\left(200^{\circ} \mathrm{C}, 240^{\circ} \mathrm{C}\right.$, and $\left.280^{\circ} \mathrm{C}\right)$ the minimal flight times and the achieved solar system escape veloci- 


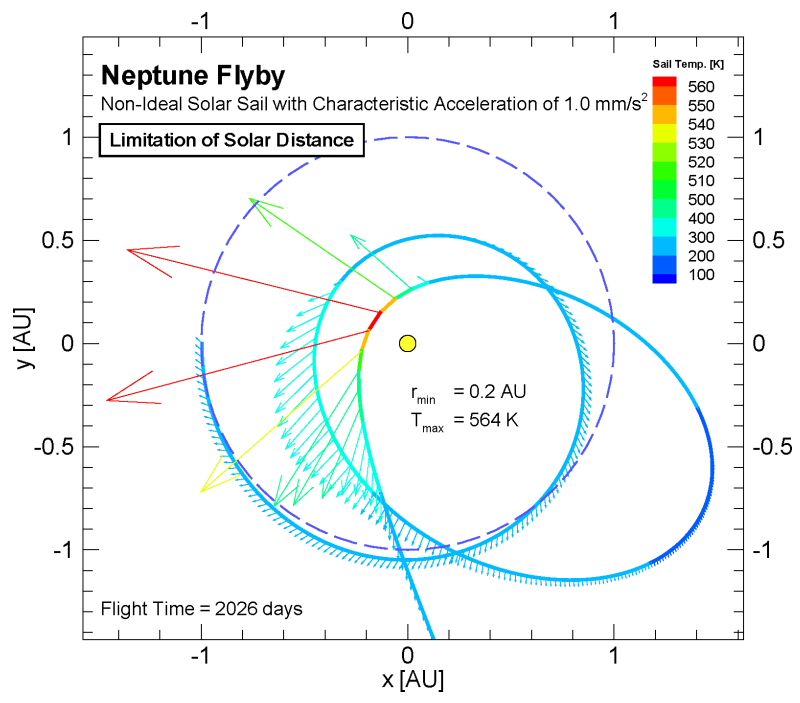

(a) Distance-limited trajectory

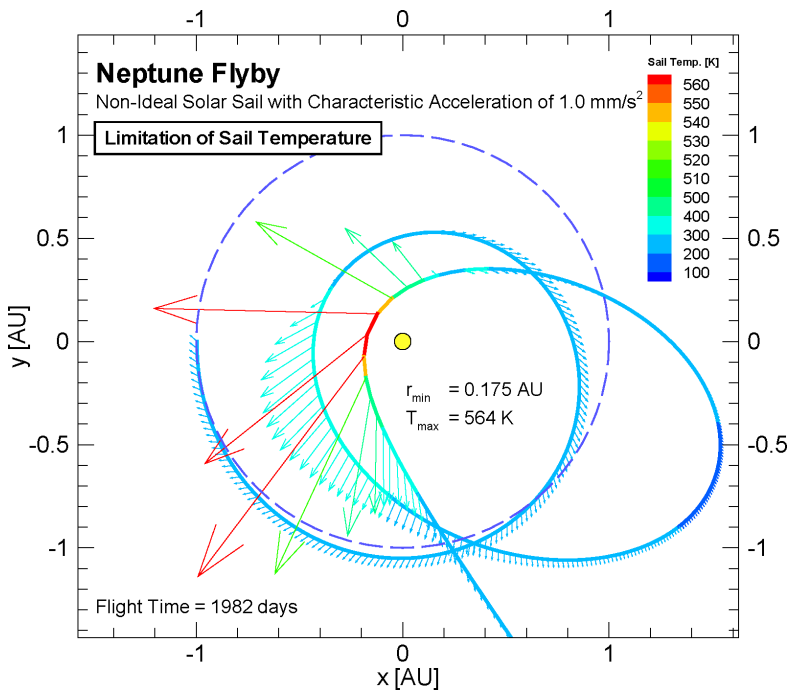

(b) Temperature-limited trajectory

Fig. 9: Optimal Neptune flyby trajectories for different optimization constraints

ties $^{5} v_{\text {esc }}$ for optimal transfers to $200 \mathrm{AU}$. Fig. 12 shows that the flight times and the solar system escape velocities depend considerably on the sail temperature limit. Both obey a potential law for all three sail temperature limits. A translation of the sail temperature limits into sail film materials can not be within the scope of this paper because the allowed maximal sail film temperature depends not only on the film material but also on the sail design (stresses, wrinkles, etc.).

\footnotetext{
${ }^{5} v_{\text {esc }}=\sqrt{ }\left(v_{f}^{2}-2 \mu / r_{f}\right)$, where $v_{f}$ is the final velocity, $r_{f}$ is the final solar distance, and $\mu$ is the sun's gravitational constant
}

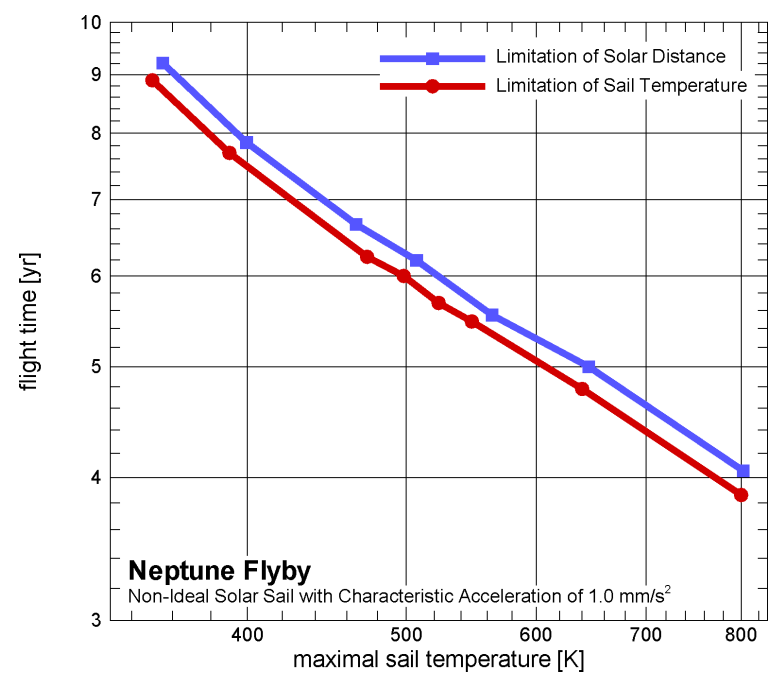

Fig. 10: Flight time over maximal sail temperature for distance-limited and temperature-limited optimal trajectories

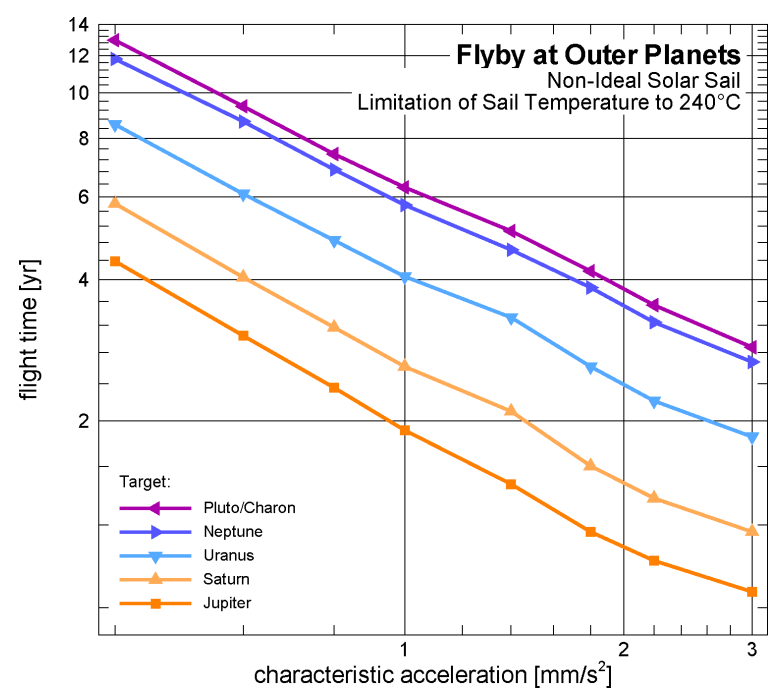

Fig. 11: Optimal flyby at the outer planets

\section{PERFORMANCE REQUIREMENTS FOR A NEAR-TERM MISSION}

Because solar sail technology has not yet reached an advanced technology readiness level, some rough order assumptions have to be made concerning the available near-term technology. Based on the experience gained at DLR in recent years from solar sail mission studies [11] and from the DLR/ESA ground deployment of a $(20 \mathrm{~m})^{2}$ breadboard sail in 1999 [12], the author considers a sail size of $(100 \mathrm{~m})^{2}$ with a sail assembly loading of $10 \mathrm{~g} / \mathrm{m}^{2}$ (sail film + booms + deployment module) as a realistic - however very challenging - baseline for a first mission to the outer solar system. 

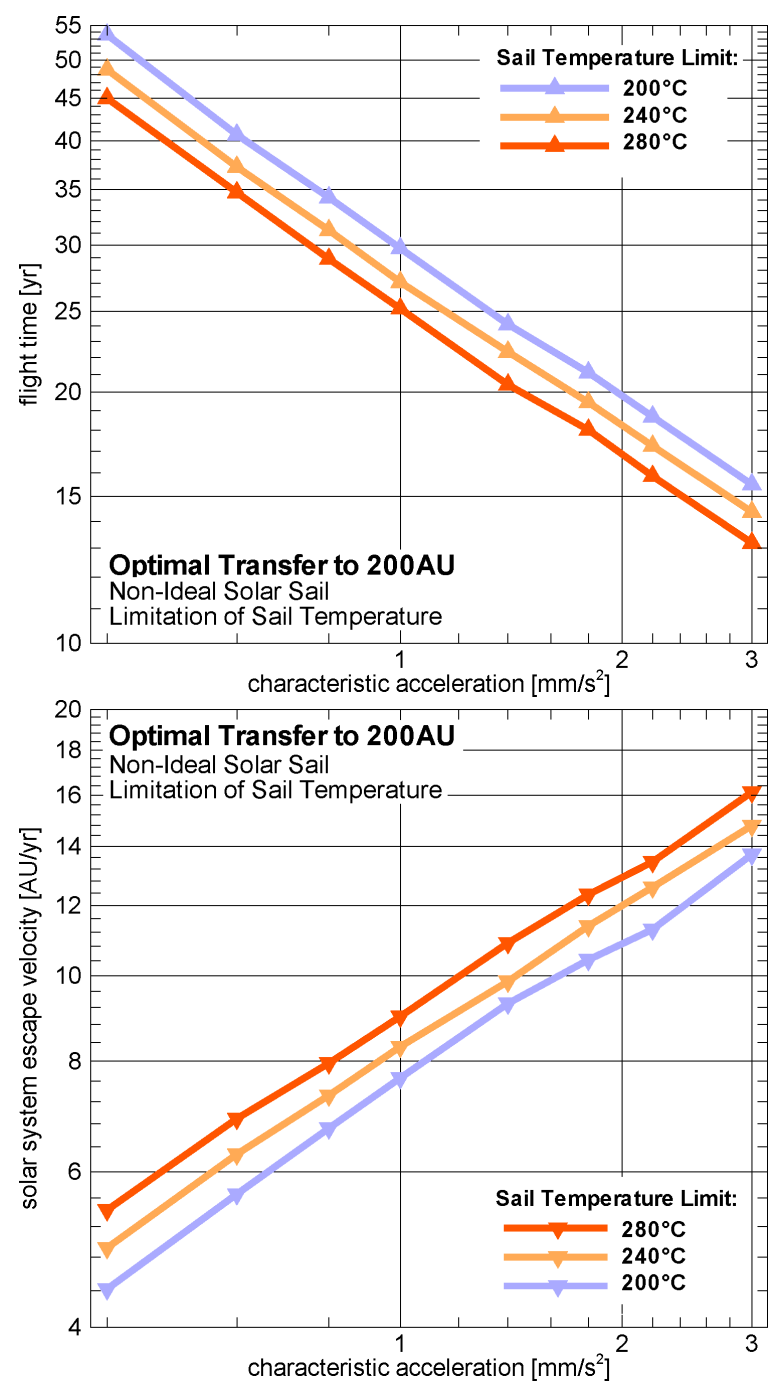

Fig. 12: Optimal transfer to $200 \mathrm{AU}$

For a square sail, equation (7) gives

$$
a_{c}=\frac{P_{\mathrm{eff}, 0}}{\sigma_{\mathrm{SA}}+m_{\mathrm{PL}} / s^{2}}
$$

One can see that the characteristic acceleration depends on three design parameters, the sail assembly loading $\sigma_{\mathrm{SA}}$, the payload mass $m_{\mathrm{PL}}$, and the sail side length $s$ (or sail area $s^{2}$ ), defining a threedimensional solar sailcraft design space. Figs. 13 and 14 show parametric sections of this design space for a fixed $\sigma_{\mathrm{SA}}=10 \mathrm{~g} / \mathrm{m}^{2}$ and for a fixed $s=100 \mathrm{~m}$, respectively. One can read from Fig. 13 that a maximal characteristic acceleration of $0.829 \mathrm{~mm} / \mathrm{s}^{2}$ can be achieved without any payload. For a lower characteristic acceleration, some payload can be accommodated, depending on the sail size. To achieve $a_{c}>0.829 \mathrm{~mm} / \mathrm{s}^{2}$, the sail assembly loading has to be reduced below $10 \mathrm{~g} / \mathrm{m}^{2}$ (Fig. 14).

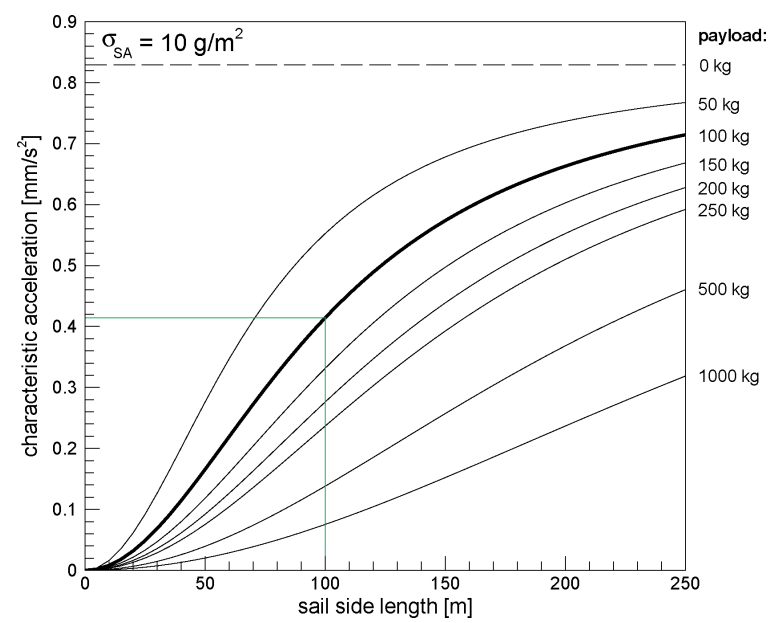

Fig. 13: Characteristic acceleration as a function of $s$ and $m_{\mathrm{PL}}$ for $\sigma_{\mathrm{SA}}=10 \mathrm{~g} / \mathrm{m}^{2}$

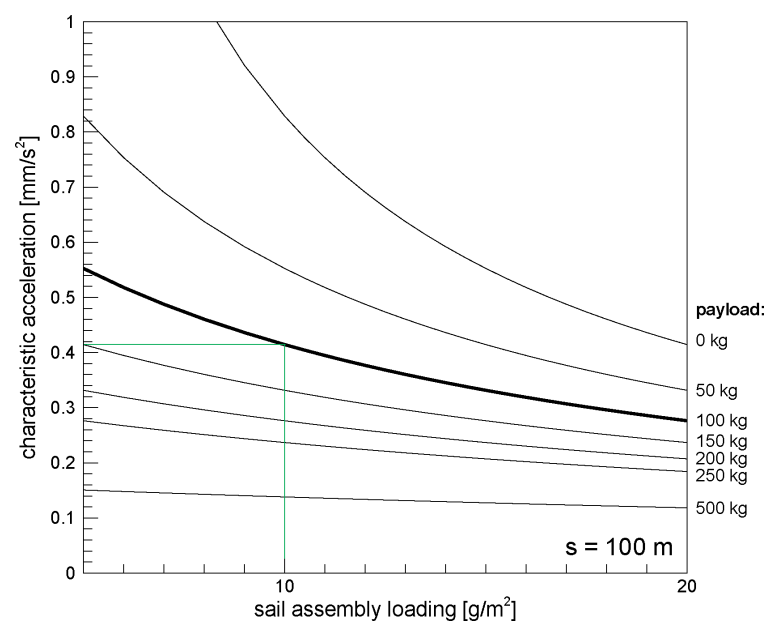

Fig. 14: Characteristic acceleration as a function of $\sigma_{\mathrm{SA}}$ and $m_{\mathrm{PL}}$ for $s=100 \mathrm{~m}$

For an exemplary payload of $m_{\mathrm{PL}}=100 \mathrm{~kg}$, a characteristic acceleration of $0.4144 \mathrm{~mm} / \mathrm{s}^{2}$ could be achieved (see Fig. 13). According to Fig. 11, this is sufficient to reach the inner EDGEWORTHKUIPER-belt within less than 13 years. For $a_{c}=$ $0.4144 \mathrm{~mm} / \mathrm{s}^{2}$, Fig. 15 shows the trade-off between sail size and sail assembly loading for various values of $m_{\mathrm{PL}}$. One can see that for $m_{\mathrm{PL}}=100 \mathrm{~kg}$ a reduction of the sail assembly loading yields only a small decrease in sail size, whereas an increase in the sail assembly loading requires a much larger solar sail. For $\sigma_{\mathrm{SA}} \gtrsim 19.5 \mathrm{~g} / \mathrm{m}^{2}$, the sail becomes to massive to achieve $a_{c}=0.4144 \mathrm{~mm} / \mathrm{s}^{2}$ even without any payload. 


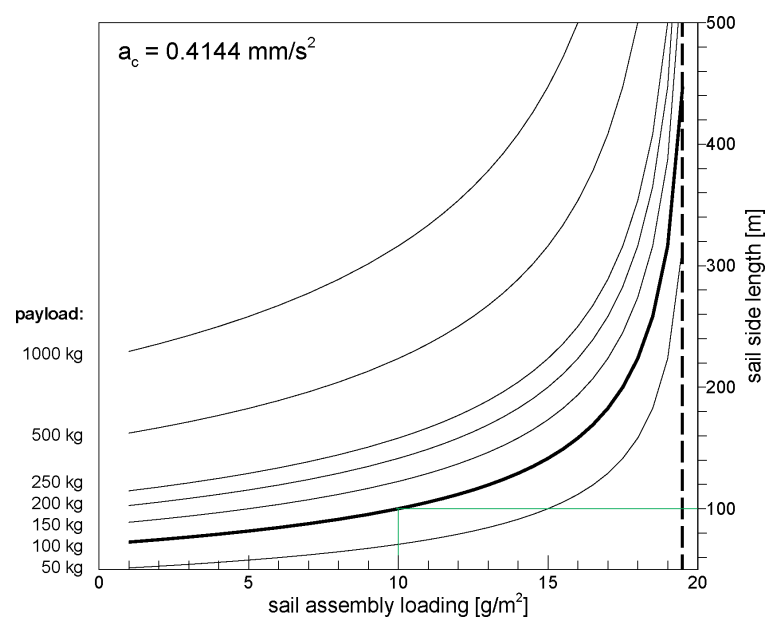

Fig. 15: Sail side length that is required to achieve $a_{c}=0.4144 \mathrm{~mm} / \mathrm{s}^{2}$ as a function of $\sigma_{\mathrm{SA}}$ and $m_{\mathrm{PL}}$

\section{SUMMARY AND CONCLUSIONS}

The material presented within this paper provides trajectory and performance trade-offs for missions to the outer solar system and into near interstellar space (200 AU). Minimal flight times have been given for different solar sail performance levels. For the 200 AU-mission, also different maximal sail temperatures have been investigated and the achieved solar system escape velocities have been given. To the authors knowledge, all previous trajectory analyses for solar system escape missions have been carried out for ideal high-performance sails. Within this paper, optimal trajectories have been presented also for non-ideal solar sails and for near/mediumterm solar sails with a relatively moderate performance. It was shown that a thorough trajectory analysis must consider the non-ideal reflectivity of the solar sail, which yields minimal flight times that are about $5 \%$ longer than those of ideal sails. To the authors knowledge, all previous trajectory analyses for solar system escape missions constrain the minimal solar distance to enforce that some sail temperature limit will not be exceeded during the closest solar approach. The trajectories calculated within this paper demonstrate that about $5 \%$ faster trajectories can be obtained, if not the allowed minimal solar distance but the allowed maximal sail temperature is directly used as an optimization constraint. A near/medium-term $(100 \mathrm{~m})^{2}$ solar sail with a specific mass of $10 \mathrm{~g} / \mathrm{m}^{2}$ would be able to transport a payload of $100 \mathrm{~kg}$ to Uranus within less than 10 years or to the inner EDGEWORTH-KUIPER belt within less than 13 years.

\section{References}

[1] C. G. Sauer. Optimum solar-sail interplanetary trajectories. San Diego, USA, August 1976. AIAA/AAS Astrodynamics Conference. 76-792.

[2] M. Leipold and O. Wagner. 'Solar photonic assist' trajectory design fo solar sail missions to the outer solar system and beyond. Greenbelt, USA, May 1998. AAS/GSFC $13^{\text {th }}$ International Symposium on Space Flight Dynamics.

[3] M. Leipold. To the sun and Pluto with solar sails and micro-sciencecraft. Acta Astronautica, 45(49):549-555, 1999.

[4] C. G. Sauer. Solar sail trajectories for solar polar and interstellar probe missions. In K. C. Howell, F. R. Hoots, and B. Kaufman, editors, Astrodynamics 1999, volume 103 of Advances in the Astronautical Sciences, pages 547-562. Univelt, Inc., 2000.

[5] B. Dachwald. Low-Thrust Trajectory Optimization and Interplanetary Mission Analysis Using Evolutionary Neurocontrol. Doctoral thesis, Universität der Bundeswehr München; Fakultät für Luft- und Raumfahrttechnik, 2004.

[6] B. Dachwald. Optimization of interplanetary solar sailcraft trajectories using evolutionary neurocontrol. Journal of Guidance, Control, and Dynamics, 27(1):66-72.

[7] B. Dachwald. Evolutionary neurocontrol: A smart method for global optimization of lowthrust trajectories. Providence, USA, August 2004. AIAA/AAS Astrodynamics Specialist Conference. AIAA-2004-5405.

[8] J. L. Wright. Space Sailing. Gordon and Breach Science Publishers, Philadelphia, 1992.

[9] C. R. McInnes. Solar Sailing. Technology, Dynamics and Mission Applications. Springer-Praxis Series in Space Science and Technology. SpringerPraxis, Berlin, Heidelberg, New York, Chicester, 1999.

[10] B. Dachwald. Minimum transfer times for nonperfectly reflecting solar sailcraft. Journal of Spacecraft and Rockets, 41(4):693-695.

[11] W. Seboldt and B. Dachwald. Solar sail (WP 0180). Technical report, ESA Propulsion 2000 Program (Phase II), 2003. Final Report Issue 1.

[12] W. Seboldt, M. Leipold, M. Rezazad, L. Herbeck, W. Unkenbold, D. Kassing, and M. Eiden. Groundbased demonstration of solar sail technology. Rio de Janeiro, Brazil, 2000. $51^{\text {st }}$ International Astronautical Congress. IAF-00-S.6.11. 